\title{
The Study of IOT Technology Based on Cloud Computing
}

\author{
Fangyun Zhao ${ }^{1, *}$, Yun Lu ${ }^{2}$ and Mingfu Zhang ${ }^{1}$ \\ ${ }^{1}$ Guizhou University of Engineering Science, School of Information Engineering, Guizhou, Bijie, 551700, China \\ ${ }^{2}$ School of Software Beihang University, Beijing, Haidian, 100191, China
}

\begin{abstract}
This paper studied the cutting-edge technology of Internet of Things (IOT) based on cloud computing. The basic ideas and methods of the combination of cloud computing and the IOT are described is this paper. By combining the cloud computing and the IOT, we can design the function modules of intelligent water resources management system. To describe the reasonableness of the combination of cloud computing and the IOT, the problems about the development of this technology are summarized, and ideas to solve security problems are developed. To ensure the efficiency of cloud computing, clustering algorithms in transport layer has been introduced, which can reduce the loss of data. We classified the data by different security levels in application layer and made the different degrees of protection with different data. Considering three-layer model of the database structures, a ploy of permissions allocation is designed. In this ploy, every allocation of permission needs a complete authentication, which can avoid the security issues caused by unauthorized users gaining unauthorized privileges in the traditional system. Besides, the support of the secondary development is essentially demanded in the development of IOT technology, and classifying the things in the IOT can make the users design it to meet this demand by themselves.
\end{abstract}

Keywords: Cloud computing, cloud platform, internet of things (IOT), network security protection.

\section{INTRODUCTION}

With the fast development of computer technology, highend technology has been applied to many fields in people's life. People are familiar with the computer intelligence, which makes their life and work convenient. Internet of Things (IOT) technology aims to build a set of networks in which each object is connected.

The IOT is the network of physical objects devices, vehicles, buildings and other items embedded with electronics, software, sensors, and network connectivity that enables these objects to collect and exchange data [1]. The IOT allows objects to be sensed and controlled remotely across existing network infrastructure [2], creating opportunities for more direct integration of the physical world into computerbased systems, and resulting in improved efficiency, accuracy and economic benefit [3-8]. When IOT is augmented with sensors and actuators, the technology becomes an instance of the more general class of cyber-physical systems, which also encompasses technologies such as smart grids, smart homes, intelligent transportation and smart cities. Each thing is uniquely identifiable through its embedded computing system but is able to interoperate within the existing Internet infrastructure. Experts estimate that the IOT will consist of almost 50 billion objects by 2020 [9]. In the IOT, all mechanical equipment has the storage and computing power. It improves the convenience greatly and meets the needs of people who cannot imagine this before. Cloud computing

*Address correspondence to this author at the Guizhou University of Engineering Science, School of information Engineering, Guizhou, Bijie, 551700, China; Tel: 13809878688; E-mail: zhangyun@163.com technology combines parallel computing, distributed computing and grid computing. The developing of the IOT depends on high efficiency storage and computing power is the advantage of cloud computing technology. That is why cloud computing technology is the basis of the IOT [10]. The IOT technology which combines cloud computing technology collects and organizes data and information by using wireless sensor and Radio frequency identification, then transmits to the application layer of the cloud computing platform. The data can share and exchange in the application layer, so that users can control and manage the whole system [11].

IOT is a network based on global electronic product code, RFID technology, the next generation of network, mobile network, the Internet and wireless communication technology, which is developing the object to object network. The IOT will go deep into all aspects of the physical world, further strengthening the social informatization. İt greatly influences and improves people's life style, thereby improving the work efficiency. Therefore, the emergence of the IOT has caused the global communications industry and governments attention, which is showing a booming potential trend. As an emerging industry, the IOT's huge development and attractive prospects for development will promote the prosperity of the global information industry development, and bring new sources of growth in all walks of life.

\section{BASIC TECHNOLOGY}

\subsection{Ubiquitous Computing in the Next Decade}

The effort by researchers to create a human-to-human interface through technology in the late 1980s resulted in the 
creation of the ubiquitous computing discipline, whose objective is to embed technology into the background of everyday life [12]. Currently, we are in the post-PC era where smart phones and other handheld devices are changing our environment by making it more interactive as well as informative. Mark Weiser, the forefather of Ubiquitous Computing (ubicomp), defined a smart environment [13] as "the physical world that is richly and invisibly interwoven with sensors, actuators, displays, and computational elements, embedded seamlessly in the everyday objects of our lives, and connected through a continuous network".

The creation of the Internet has marked a foremost milestone towards achieving vision which enables individual devices to communicate with any other device in the world. The inter-networking reveals the potential of a seemingly endless amount of distributed computing resources and storage owned by various owners (Fig. 1).

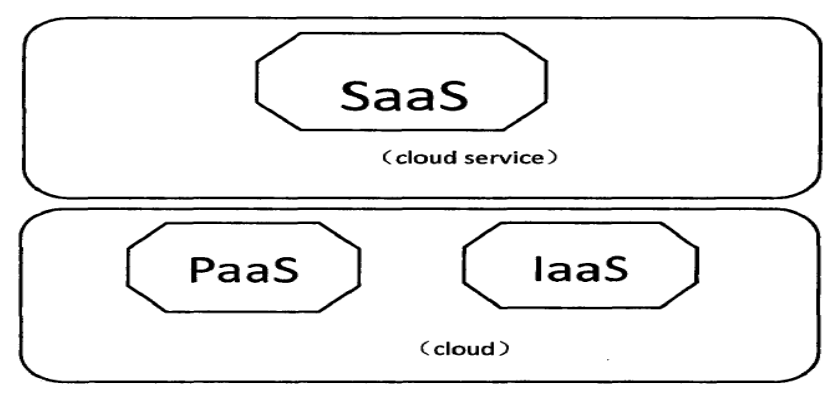

Fig. (1). Cloud computing type.

\subsection{Definitions}

According to Cluster of European research projects on the IOT [14] -'Things' are active participants in business, information and social processes where they are enabled to interact and communicate among themselves and with the environment by exchanging data and information sensed about the environment, while reacting autonomously to the real/physical world events and influencing it by running processes that trigger actions and create services with or without direct human intervention.

As human gathering centers and trade centers, cities are the signs of human civilization progress. Urbanization is an inevitable trend of urban development. The way towards urbanization is coupled with a string of social problems, which is exemplified by the low efficiency of urban management model, crowded traffic system, emergency system without actual effect, and incomplete environmental monitoring system, etc. Digital cities collect, analyze and deal with urban management problems based on various technical means such as GPS, GIS, RS, computers, network communication and so on. Significant results are obtained by the implementation of grid management and urban component management in urban order, traffic, sanitation and planning. Smart cities' information systems must have enhanced computing power, sensory ability and data application. The development of cloud computing, IOT and semantic net is the matter of feasibility to the construction of smart cities. The research of cloud computing, IOT and the Semantic Web application infrastructure and key technology has important theoretical significance and practical value (Fig. 2).

IOT involves a lot of technologies and standardization organizations; its industrial chain is huge and wide; and its development needs the government's policy support, mutual cooperation and exchange between enterprises, and the joint efforts of the organization. Analyzing the development of the IOT in my country, will find that telecom operators in its industry chain are shouldering the integrated industrial chain upstream and downstream, and playing the facilitator role in the development of the IOT. By using its perfect network resources and large customer to establish the basis of Internet information transmission bearing body, the industry uses it to call for pushing the networking technology and business innovation. To play the above role, operators need to establish an open platform for the IOT operation management [15], such as intelligent pipe platform operation. On the one hand, we are expanding their space for income, by attracting a large number of developers to develop business on this platform, aggregation of various kinds of applications, providing centralized services, thereby further improving the operator's brand image. Based on the above reasons, this article's research has important theoretical value and practical significance (Fig. 3).

Currently, the information systems used in most companies are built by themselves, and can only be used for the internal. Information between companies cannot be shared effectively. The upfront and maintenance cost is also very high. To solve this problem, this study proposes a method of the information management system designed based on cloud computing. This method takes full advantages of cloud computing, reaches the goals that share the equipments, information and services, and can help enterprises reduce cost and share resources [16].

\section{IOT ELEMENTS}

We present a taxonomy that will aid in defining the components required for the IOT from a high level perspective. Specific taxonomies of each component can be found elsewhere. We analyzed the IOT's three-layer architecture perception layer app [17]. There are three IOT components which enable seamless: (a) Hardware-made up of sensors, actuators and embedded communication hardware; (b) Middleware-on demand storage and computing tools for data analytics; and (c) Presentation-novel easy to understand visualization and interpretation tools which can be widely accessed on different platforms and which can be designed for different applications. In this section, we discussed a few enabling technologies in these categories which will make up the three components stated above as shown in Table $\mathbf{1}$. 


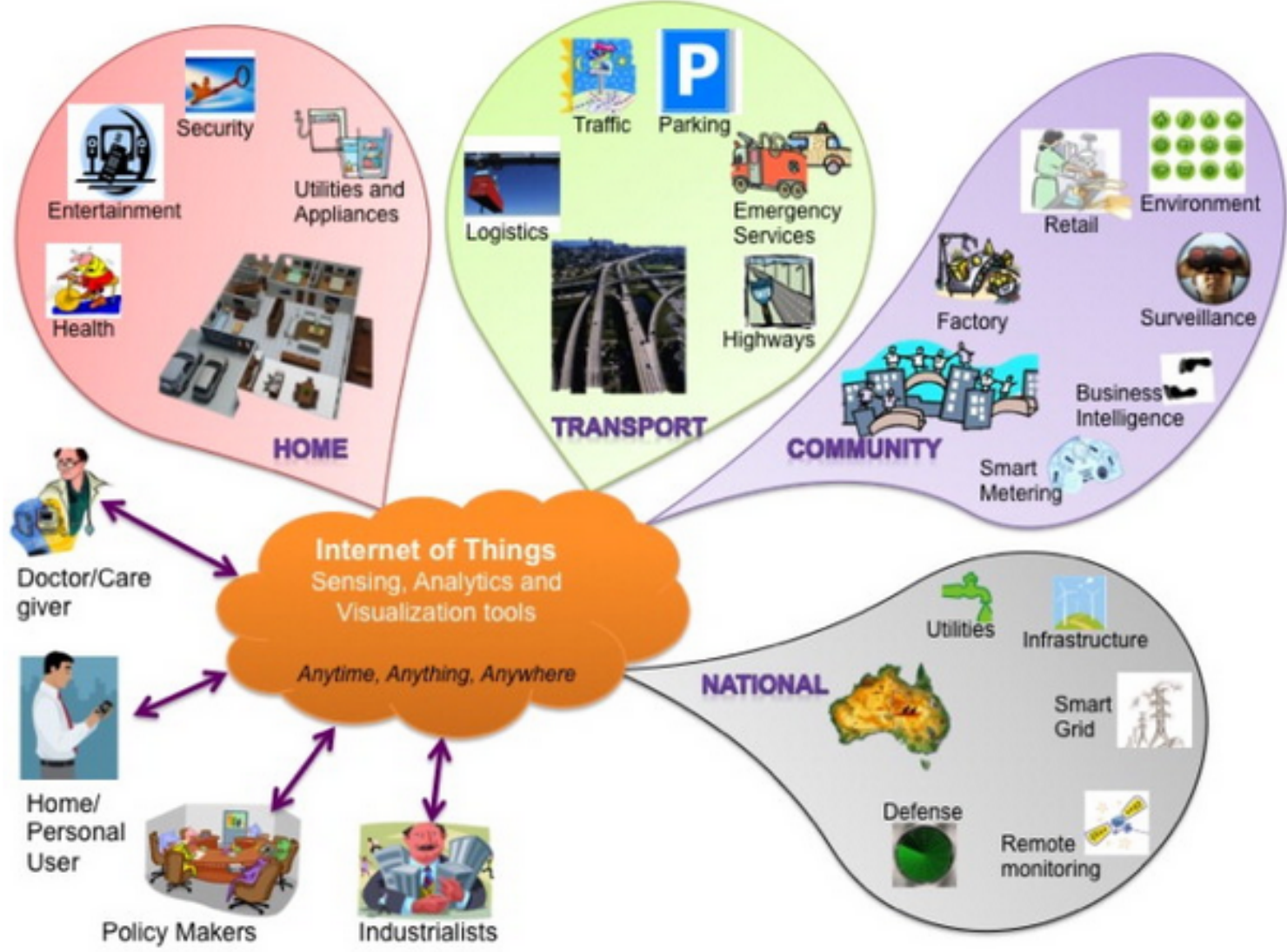

Fig. (2). IOT schematic showing the end users and application areas based on data.

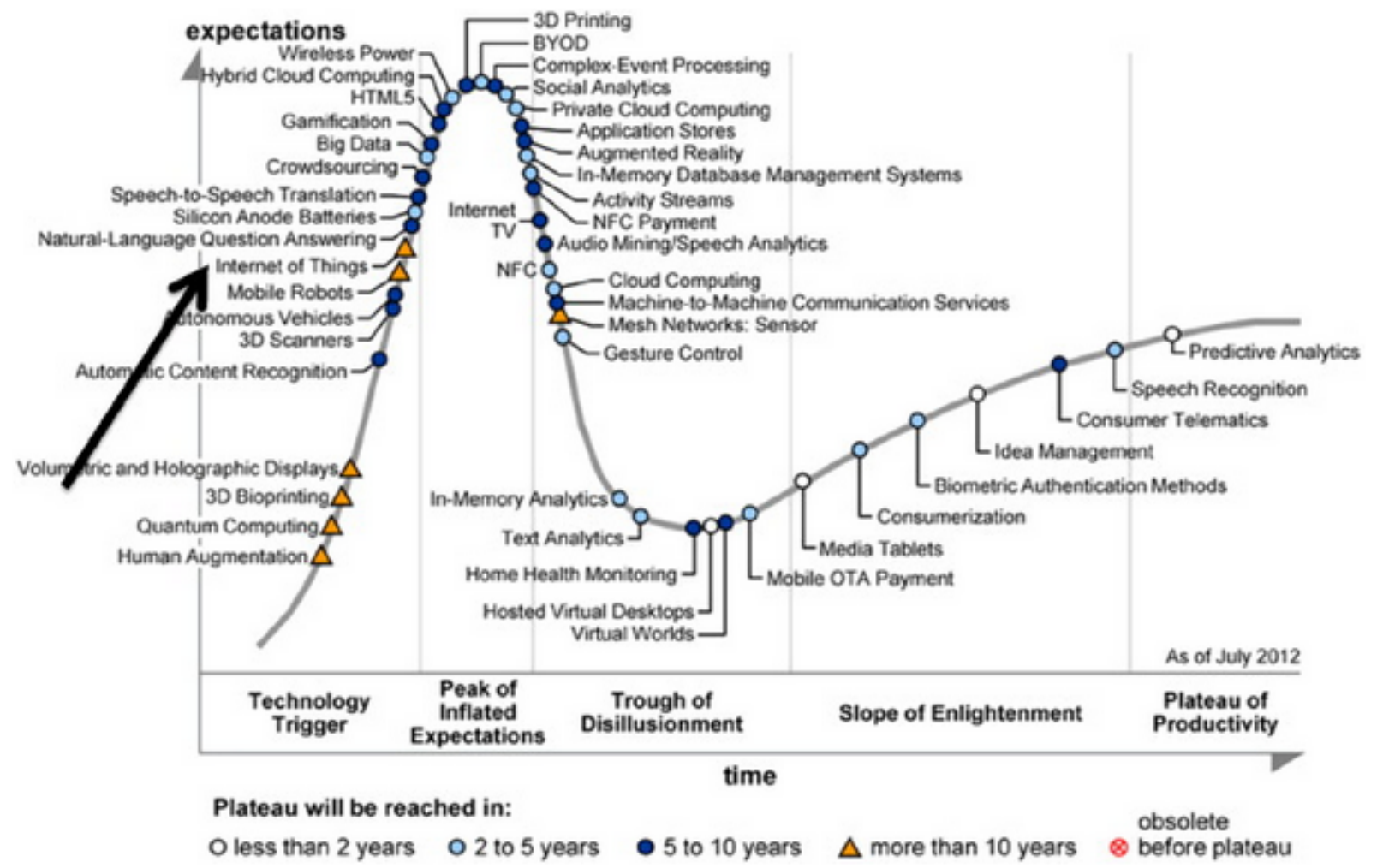

Fig. (3). Gartner 2012 hype cycle of emerging technologies gartner Inc. 
Table 1. Smart environment application domains.

\begin{tabular}{|c|c|c|c|c|c|c|}
\hline $\begin{array}{c}\text { Smart home/offi } \\
\text { ce }\end{array}$ & $\begin{array}{l}\text { Smart } \\
\text { retail }\end{array}$ & Smart city & $\begin{array}{c}\text { Smart agriculture/ } \\
\text { forest }\end{array}$ & Smart water & $\begin{array}{c}\text { Smart transporta- } \\
\text { tion }\end{array}$ & \\
\hline Network size & Small & Small & Medium & Medium/large & Large & Large \\
\hline Users & $\begin{array}{l}\text { Very few, } \\
\text { fami- } \\
\text { ly members }\end{array}$ & $\begin{array}{c}\text { Few, communit } \\
\text { y level }\end{array}$ & $\begin{array}{l}\text { Many, policy make } \\
\text { rs, general public }\end{array}$ & $\begin{array}{c}\text { Few, landowners, } \\
\text { policy makers }\end{array}$ & Few, government & Large, general public \\
\hline Energy & $\begin{array}{l}\text { Rechargea- } \\
\text { ble battery }\end{array}$ & $\begin{array}{c}\text { Rechargeable } \\
\text { battery }\end{array}$ & $\begin{array}{l}\text { Rechargea- } \\
\text { ble battery, ener- } \\
\text { gy harvesting }\end{array}$ & Energy harvesting & Energy harvesting & $\begin{array}{c}\text { Rechargeable battery, } \\
\text { Energy harvesting }\end{array}$ \\
\hline $\begin{array}{l}\text { Internet connec- } \\
\text { tivity }\end{array}$ & $\begin{array}{c}\text { Wi- } \\
\text { Fi, 3G, } 4 \mathrm{G} \\
\text { LTE back- } \\
\text { bone }\end{array}$ & $\begin{array}{c}\text { Wi- } \\
\text { Fi, 3G, 4G LTE } \\
\text { backbone }\end{array}$ & $\begin{array}{c}\text { Wi-Fi, 3G, 4G LTE } \\
\text { backbone }\end{array}$ & $\begin{array}{l}\text { Wi-Fi, satellite com- } \\
\text { munication }\end{array}$ & $\begin{array}{l}\text { Satel- } \\
\text { lite communication, } \\
\text { microwave links }\end{array}$ & $\begin{array}{l}\text { Wi-Fi, satellite com- } \\
\text { munication }\end{array}$ \\
\hline Data management & $\begin{array}{l}\text { Local } \\
\text { server }\end{array}$ & Local server & Shared server & $\begin{array}{l}\text { Local server, } \\
\text { shared server }\end{array}$ & Shared server & Shared server \\
\hline IOT devices & $\begin{array}{l}\text { RFID, } \\
\text { WSN }\end{array}$ & RFID, WSN & RFID, WSN & WSN & Single sensors & $\begin{array}{l}\text { RFID, WSN, single } \\
\text { sensors }\end{array}$ \\
\hline $\begin{array}{c}\text { Bandwidth re- } \\
\text { quirement }\end{array}$ & Small & Small & Large & Medium & Medium & Medium/large \\
\hline Example testbeds & $\begin{array}{l}\text { Aware } \\
\text { home }\end{array}$ & $\begin{array}{l}\text { SAP future retai } \\
1 \text { center }[8]\end{array}$ & $\begin{array}{c}\text { Smart Santander, } \\
\text { citySense [9] }\end{array}$ & SiSViA [10] & & \\
\hline
\end{tabular}

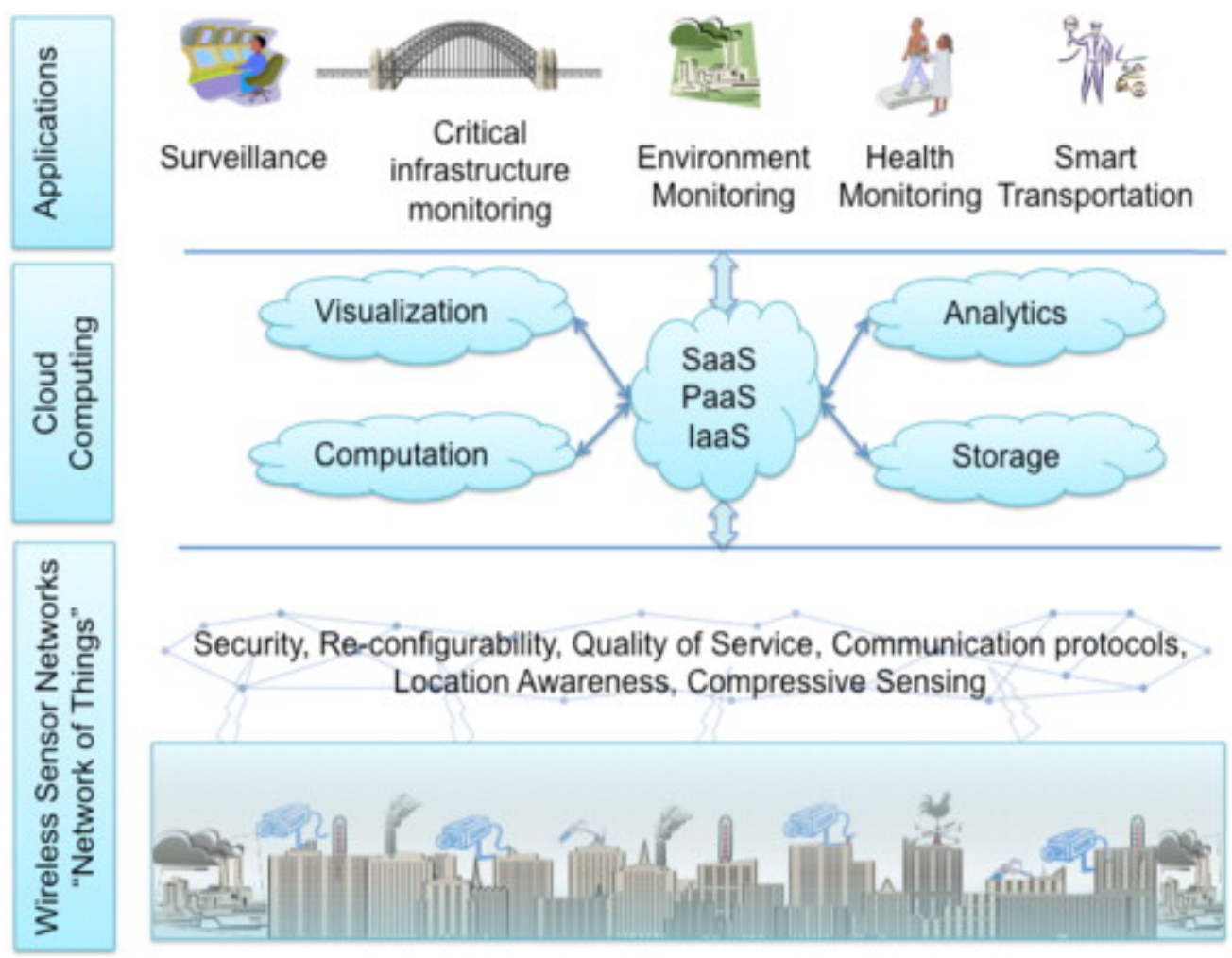

Fig. (4). Conceptual IOT framework with cloud computing at the center. 


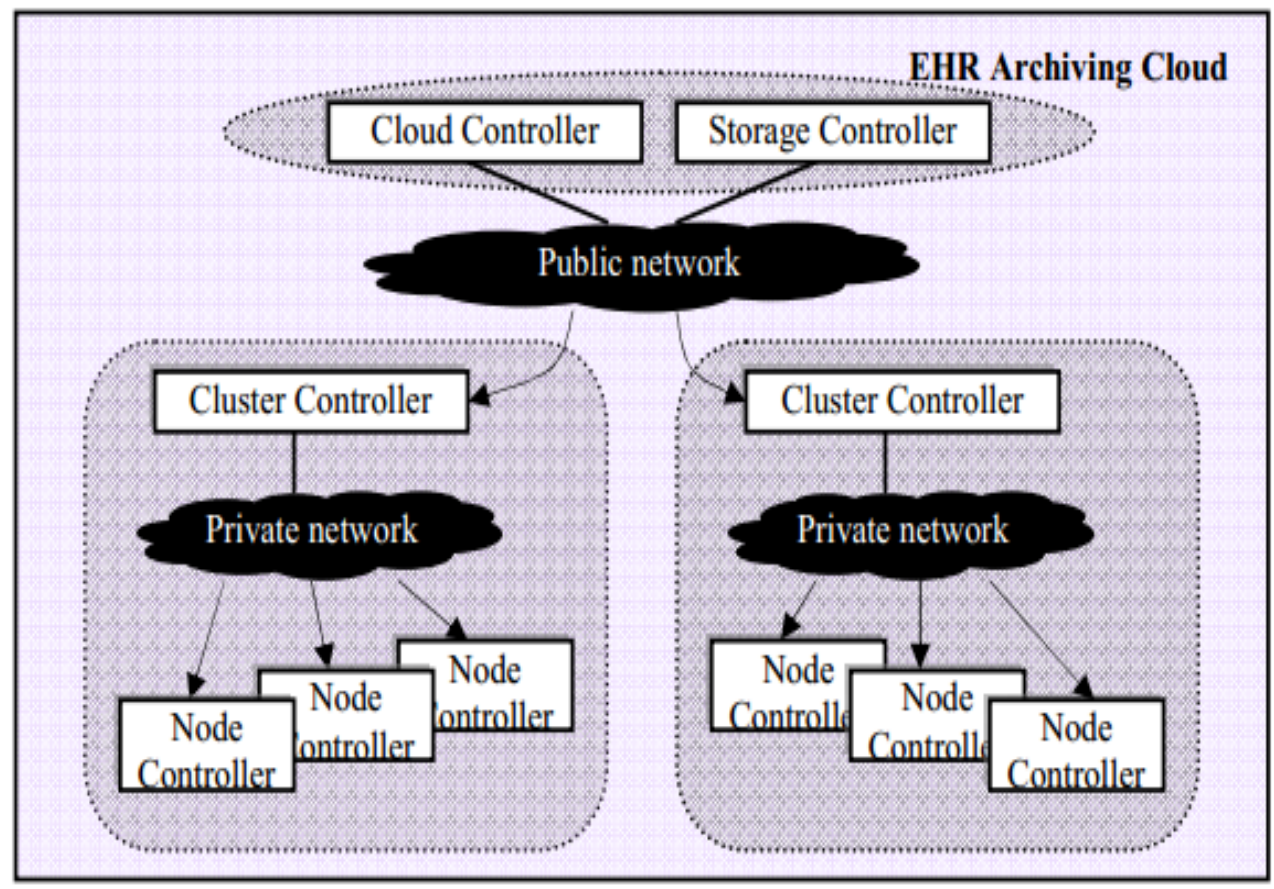

Fig. (5). Elastic archiving based on EUCALYPTUS cloud.

\subsection{Wireless Sensor Networks (WSN)}

Recent technological advances in low power integrated circuits and wireless communications have made available efficient, low cost, low power miniature devices for use in remote sensing applications. The combination of these factors has improved the viability of utilizing a sensor network consisting of a large number of intelligent sensors, enabling the collection, processing, analysis and dissemination of valuable information, gathered in a variety of environments. Active RFID is nearly the same as the lower end WSN nodes with limited processing capability and storage. The scientific challenges that must be overcome in order to realize the enormous potential of WSNs are substantial and multidisciplinary in nature. Sensor data are shared among sensor nodes and sent to a distributed or centralized system for analytics (Fig. 4).

\subsection{Data Storage and Analytics}

One of the most important outcomes of this emerging field is the creation of an unprecedented amount of data storage [18]. Ownership and expiry of the data became critical issues. The internet consumes up to $5 \%$ of the total energy generated today and with these types of demands, it is sure to go up even further. Hence, data centers that run on harvested energy and are centralized will ensure energy efficiency as well as reliability. The data have to be stored and used intelligently for smart monitoring and actuation. It is important to develop artificial intelligence algorithms which could be centralized or distributed based on the need. Novel fusion algorithms need to be developed to make sense of the data collected. State-of-the-art non-linear, temporal machine learning methods based on evolutionary algorithms, genetic algorithms, neural networks, and other artificial intelligence techniques are necessary to achieve automated decision mak- ing. These systems show characteristics such as interoperability, integration and adaptive communications. They also have a modular architecture both in terms of hardware system design as well as software development and are usually very well-suited for IOT applications. More importantly, a centralized infrastructure to support storage and analytics is required. This forms the IOT middleware layer and there are numerous challenges involved which are discussed in future sections. As of 2012, cloud based storage solutions are becoming increasingly popular and in the years ahead, cloud based analytics and visualization platforms are foreseen.

In EUCALYPTUS, the Virtual Machines are implemented through the Xen hypervisor, while the high-level system components are implemented as a standalone Web service [19]. The four major high-level components are explained as follows (Fig. 5): Node Controller controls the execution, inspection, and terminating of VM instances on the host where it runs; Cluster Controller gathers information about and schedules VM execution on specific node controllers, as well as manages virtual instance network; Storage Controller is a put/get storage service that implements Amazon's S3 interface, providing a mechanism for storing and accessing VM images and user data; and Cloud Controller is the entrypoint into the cloud for users and administrators. It queries node managers for information about resources, makes high level scheduling decisions, and implements them by making requests to cluster controllers. Under the framework of EUCALYPTUS, EHRs system is able to elastically start, control, access, and terminate entire Virtual Machines using an emulation of Amazon EC2's SOAP and "Query" interfaces [20]. When more data is coming and requires more space, the system dynamically starts more virtual machines with more storage. While the dynamic archiving requirement meets, the system may not be rooted and user can't perceive it. 


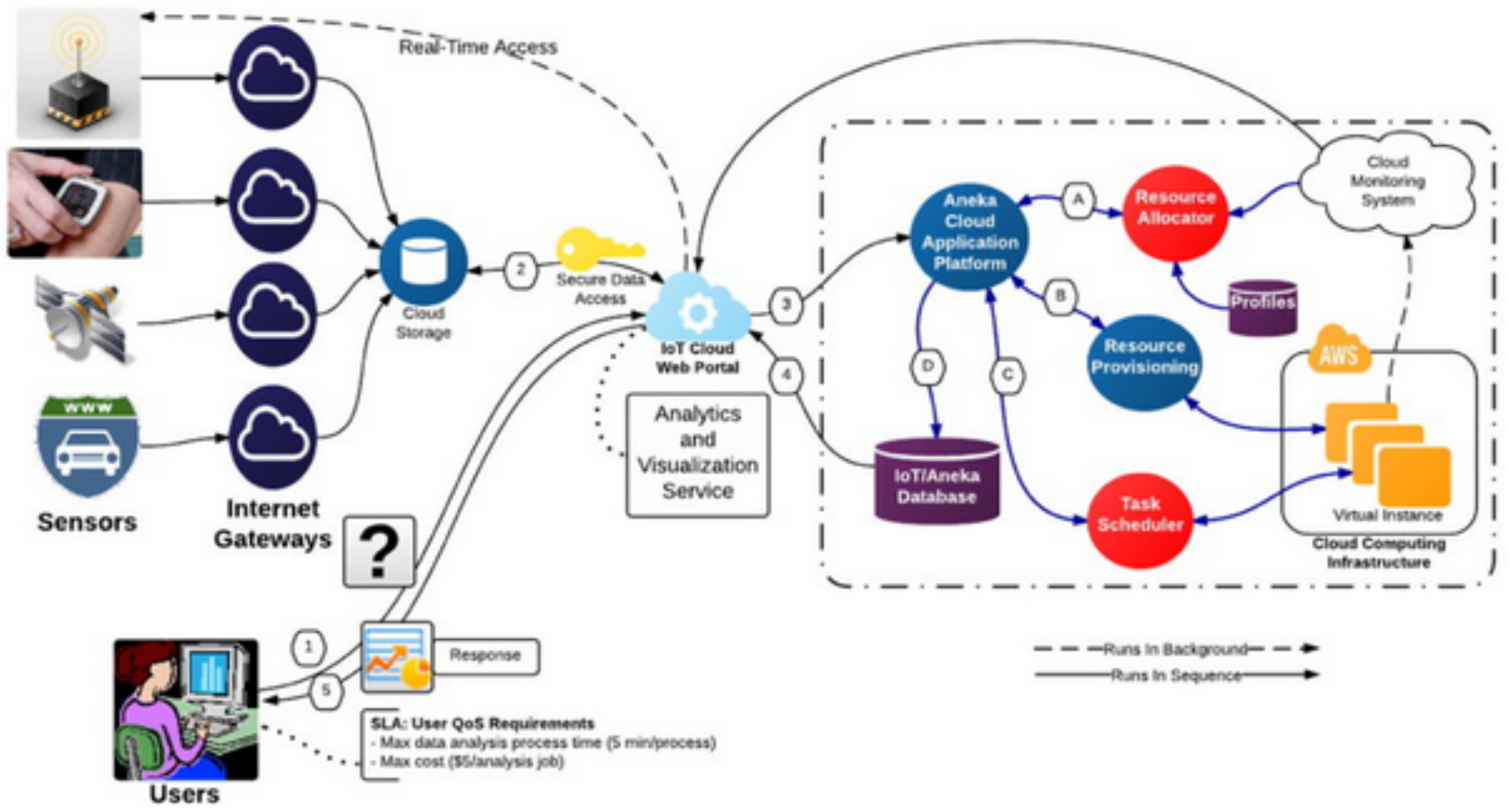

Fig. (6). A model of end-to-end interaction between various stakeholders in cloud centric IOT framework.

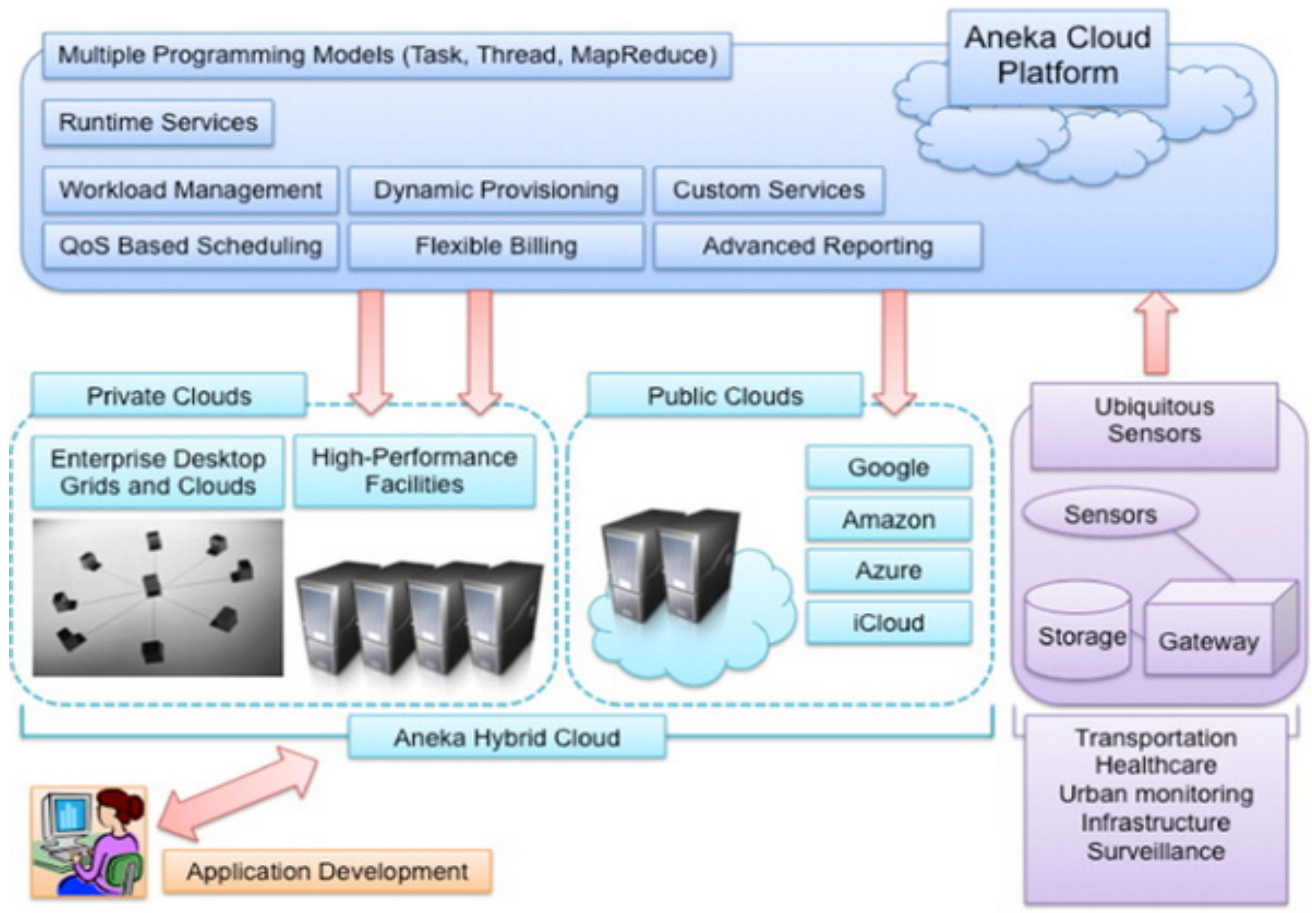

Fig. (7). Overview of aneka within IOT architecture.

The proposed cloud centric vision comprises a flexible and open architecture that is user centric and enables different players to interact in the IOT framework. It allows interaction in a manner suitable for their own requirements, rather than the IOT being thrust upon them (Fig. 6).

\section{APPLICATION}

There are several application domains which will be impacted by the emerging IOT. The applications can be classi- fied based on the type of network availability, coverage, scale, heterogeneity, repeatability, user involvement and impact. We categorize the applications into four application domains: (1) Personal and Home; (2) Enterprise; (3) Utilities; and (4) Mobile. This is depicted in Fig. (1), which represents: Personal and Home IOT at the scale of an individual or home; Enterprise IOT at the scale of a community; Utility IOT at a national or regional scale and Mobile IOT which is usually spread across other domains mainly due to the nature 


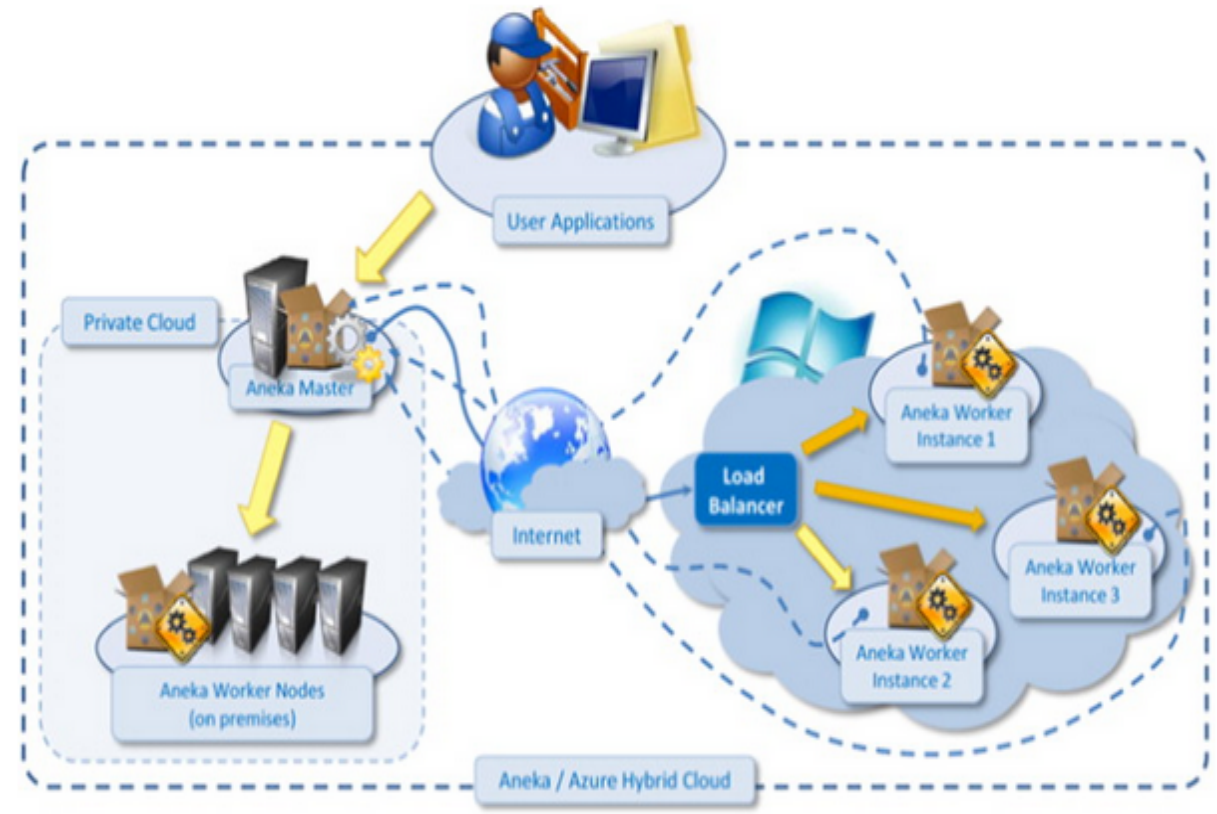

Fig. (8). Schematic of aneka/azure interaction for data analytics application.

of connectivity and scale. There is a huge crossover in applications and the use of data between domains. For instance, the Personal and Home IOT produces electricity usage data in the house and makes it available to the electricity (utility) company which can in turn optimize the supply and demand in the Utility IOT. The internet enables sharing of data between different service providers in a seamless manner creating multiple business opportunities. A few typical applications in each domain are given.

The Aneka scheduler is responsible for assigning each resource to a task in an application for execution based on user QoS parameters and the overall cost for the service provider. Depending on the computation and data requirements of each Sensor Application, it directs the dynamic resource provisioning component to instantiate or terminate a specified number of computing, storage, and network resources while maintaining a queue of tasks to be scheduled. This logic is embedded as multi-objective application scheduling algorithms. The scheduler is able to manage resource failures by reallocating those tasks to other suitable cloud resources.

The Dynamic Resource Provisioning component implements the logic for provisioning and managing virtualized resources in the private and public cloud computing environments based on the resource requirements as directed by the application scheduler. This is achieved by dynamically negotiating with the Cloud Infrastructure as a Service (IaaS) providers for the right kind of resource for a certain time and cost by taking into account the past execution history of applications and budget availability. This decision is made at runtime, when SaaS applications continuously send requests to the Aneka cloud platform.

As discussed earlier, to realize the vision, tools and data are required to be shared between application developers to create new apps. There are two major hurdles in such an implementation. Firstly, interaction between clouds becomes critical which is addressed by Aneka in the InterCloud mod- el. Aneka support for the InterCloud model enables the creation of a hybrid Cloud computing environment that combines the resources of private and public Clouds. That is, whenever a private Cloud is unable to meet application of QoS requirements, Aneka leases extra capability from a public Cloud to ensure that the application is able to execute within a specified deadline in a seamless manner. Secondly, data analytics and artificial intelligence tools are computationally demanding, which requires huge resources. For data analytics and artificial intelligence tools, the Aneka task programming model provides the ability of expressing applications as a collection of independent tasks. Each task can perform different operations, or the same operation on different data, and can be executed in any order by the runtime environment. In order to demonstrate this, we have used a scenario where there are multiple analytics algorithms and multiple data sources. A schematic of the interaction between Aneka and Azure is given in Fig. (7), where Aneka Worker Containers are deployed as instances of Azure Worker Role. The Aneka Master Container will be deployed in the on-premises private cloud, while Aneka Worker Containers will be run as instances of Microsoft Azure Worker Role. As shown in Fig. (8), there are two types of Microsoft Azure Worker Roles used. These are the Aneka Worker Role and Message Proxy Role. In this case, one instance of the Message Proxy Role and at least one instance of the Aneka Worker Role are deployed. The maximum number of instances of the Aneka Worker Role that can be launched is limited by the subscription offer of Microsoft Azure Service that a user selects. In this deployment scenario, when a user submits an application to the Aneka Master, the job units will be scheduled by the Aneka Master by leveraging on-premises Aneka Workers, if they exist, and Aneka Worker instances on Microsoft Azure simultaneously. When Aneka Workers finish the execution of Aneka work units, they will send the results back to Aneka Master, and then Aneka Master will send the result back to the user application. 
Some open challenges are discussed based on the IOT elements presented earlier. The challenges include IOT specific challenges such as privacy, participatory sensing, data analytics, GIS based visualization and Cloud computing apart from the standard WSN challenges including architecture, energy efficiency, security, protocols, and Quality of Service. The end goal is to have Plug n' Play smart objects which can be deployed in any environment with an interoperable backbone allowing them to blend with other smart objects around them. Standardization of frequency bands and protocols plays a pivotal role in accomplishing this goal. A roadmap of key developments in IOT research in the context of pervasive applications, which includes the technology drivers and key application outcomes expected in the next decade.

\section{CONCLUSION}

Ubiquitous sensing enabled by Wireless Sensor Network (WSN) technologies cuts across many areas of modern day living. This offers the ability to measure, infer and understand environmental indicators, from delicate ecologies and natural resources to urban environments. The proliferation of these devices in a communicating-actuating network creates the Internet of Things (IOT), wherein sensors and actuators blend seamlessly with the environment around us, and the information is shared across platforms in order to develop a common operating picture (COP). Fueled by the recent adaptation to a variety of enabling wireless technologies such as RFID tags and embedded sensor and actuator nodes, the IOT has stepped out of its infancy and is the next revolutionary technology in transforming the Internet into a fully integrated Future Internet. As we move from www (static pages web) to web2 (social networking web) to web3 (ubiquitous computing web), the need for data-on-demand using sophisticated intuitive queries increases significantly. This paper presents a Cloud centric vision for worldwide implementation of IOT. The key enabling technologies and application domains that are likely to drive IOT research in the near future are discussed. A Cloud implementation using Aneka, which is based on interaction of private and public Clouds, is presented. We conclude our IOT vision by expanding on the need for convergence of WSN, the Internet and distributed computing directed at technological research community.

\section{PROJECT SUPPORT}

This work is supported by The basic research funds for the project of Southwestern University (XDJK2014C109); Guizhou science and Technology Fund Project, Department of Guizhou LH words [2014]7536.

\section{CONFLICT OF INTEREST}

The authors confirm that this article content has no conflict of interest.

\section{ACKNOWLEDGEMENTS}

Declared none.

\section{REFERENCES}

[1] G. Mulligan, "Internet of things: here now and coming soon," IEEE Internet Computing, vol. 14, no. 1, pp. 35-36, 2010.

[2] A. J. Jara, M. A. Zamora and A. F. G. Skarmeta, "An ambient assisted living system for telemedicine with detection of symptoms", In: $3^{\text {rd }}$ International Work-Conference on the Interplay Between Natural and Artificial Computation, Lecture Notes, 2009, pp. 75-84.

[3] X. M. Zhang, and J. Li, "Research on interoperability of internet of things' gateway oriented to telehealth and telemedicine," IEEE Proceeding of 2010 International Conference on Future Information Technology, vol. 2, pp. 406-410, 2010.

[4] D.Y. Fei, X. Zhao, C. Boanca, E. Hughes, O. Bai, R. Merrell, and A. Rafiq, "A biomedical sensor system for real-time monitoring of astronauts' physiological parameters during extravehicular activities," Computer in Biology and Medicine, vol. 40, pp. 635-642, 2010.

[5] The Continua Health Alliance, http://www.continuaalliance.org/

[6] R. Carroll, R. Cnossen, M. Schnell and D. Simons, "Continua: an interoperable personal healthcare ecosystem," IEEE Pervasive Computing, vol. 6, no. 4, pp. 90-94, 2007.

[7] USB Personal Healthcare Device Profile, http://www.usb.org

[8] X. M. Zhang, H. M. Wang and Q. Y. Wu, "High performance virtual distributed object," Journal of Computer Research and Development, pp. 102-107, 2000.

[9] X. M. Zhang, Q. Y. Wu, H. M. Wang and Y. Jia, "A dynamic scalable asynchronous message model based on distributed objects," Computer Engineering and Science, vol. 3, pp. 48-50, 2002.

[10] X. M. Zhang, "A semantic grid oriented to e-tourism," In: IEEE Proceeding of the $1^{\text {st }}$ International Conference on Cloud Computing (CloudCom 2009), Beijing, 2009, pp. 485-496.

\begin{tabular}{lcc}
\hline Received: May 26, 2015 & Revised: July 14, 2015 & Accepted: August 10, 2015 \\
C) Zhao et al.; Licensee Bentham Open. &
\end{tabular}

This is an open access articles licensed under the terms of the Creative Commons Attribution-Non-Commercial 4.0 International Public License (CC BY-NC 4.0) (https://creativecommons.org/licenses/by-nc/4.0/legalcode), which permits unrestricted, non-commercial use, distribution and reproduction in any medium, provided that the work is properly cited. 